\title{
Quelques observations sur le comportement de formes préimaginales de Culicidés sous l'influence de la lumière
}

\author{
Par J. CALLoT
}

L'étude de l'action de la lumière sur les formes préimaginales des moustiques a donné lieu â des observations nombreuses et dispersées quant à l'effet de l'éclairement des gites mais à peu de recherches expérimentales.

Roubaud et Colas-Belcour (1932), dans un mémoire sur Anopheles plumbeus, ont montré que les larves de cette espèce, placées dans un tube de verre éclairé à une extrémité, se portaient vers l'extrémité la moins éclairée et ont ainsi objectivé un phototropisme négatif.

Omardeen (1957) est le seul auteur qui, à notre connaissance, ait donné une représentation du comportement de larve et de nymphes placées, soit dans un champ uniformément éclairé, soit dans un gradient lumineux bien défini. Ses recherches ont porté uniquement sur Aedes aegypti à différents stades préimaginaux et étaient inspirées du travail d'Ivanova (1940), c'est-à-dire qu'il s'agissait aussi de l'étude du comportement dans un gradient thermique en lumière uniforme.

Par des histogrammes très suggestifs, résultat de mesures effectuées sur les larves et les nymphes, Omardeen montre que le phototropisme, d'abord indifférent au stade 1 et 2 larvaires, devient nettement négatif au stade 4 et chez la nymphe.

Quand nous avons commencé cette étude nous ne connaissions pas le travail d'Omardeen, qui n'avait pas encore été publié, et nous avions réalisé un montage qui, par un phénomène de convergence parfaitement compréhensible, était à peu de choses près identique à celui de cet auteur.

\section{MATERIEL ET METHODE}

L'appareil est essentiellement constitué par une gouttière placée sous la ou les sources lumineuses dont on veut étudier l'action.

La gouttière est en tôle d'aluminium, longue de $100 \mathrm{~cm}$, large de $4 \mathrm{~cm}$, profonde de $3,5 \mathrm{~cm}$. On y met de l'eau pour avoir une profondeur d'environ $2 \mathrm{~cm}$. La gouttière est surmontée par le dispositif d'éclairage placé à $35 \mathrm{~cm}$ au-dessus de la surface de l'eau. Il est 
constitué par une boîte parallélépipède de $1 \mathrm{~m}$ et dont la surface inférieure est une plaque de verre dépolie. L'éclairage est produit par des ampoules ordinaires du commerce de $60 \mathrm{~W}$ (750 lux) suspendues de façon que leur pôle inférieur soit à $5 \mathrm{~cm}$ au-dessus de la plaque dépolie. Pour un éclairage uniforme on place une ampoule à chaque extrémité et deux autres au centre à distances égales les unes des autres. Pour déterminer un gradient on place deux ampoules de $60 \mathrm{~W}$, à une extrémité de la gouttière (au-dessus de la case 1). Pour éviter l'échauffement, un cristallisoir d'eau est interposé. Les mesures photométriques montrent qu'avec ce système assez simple on a un éclairage relativement uniforme ou un gradient régulier et que l'échauffement de l'eau est négligeable.

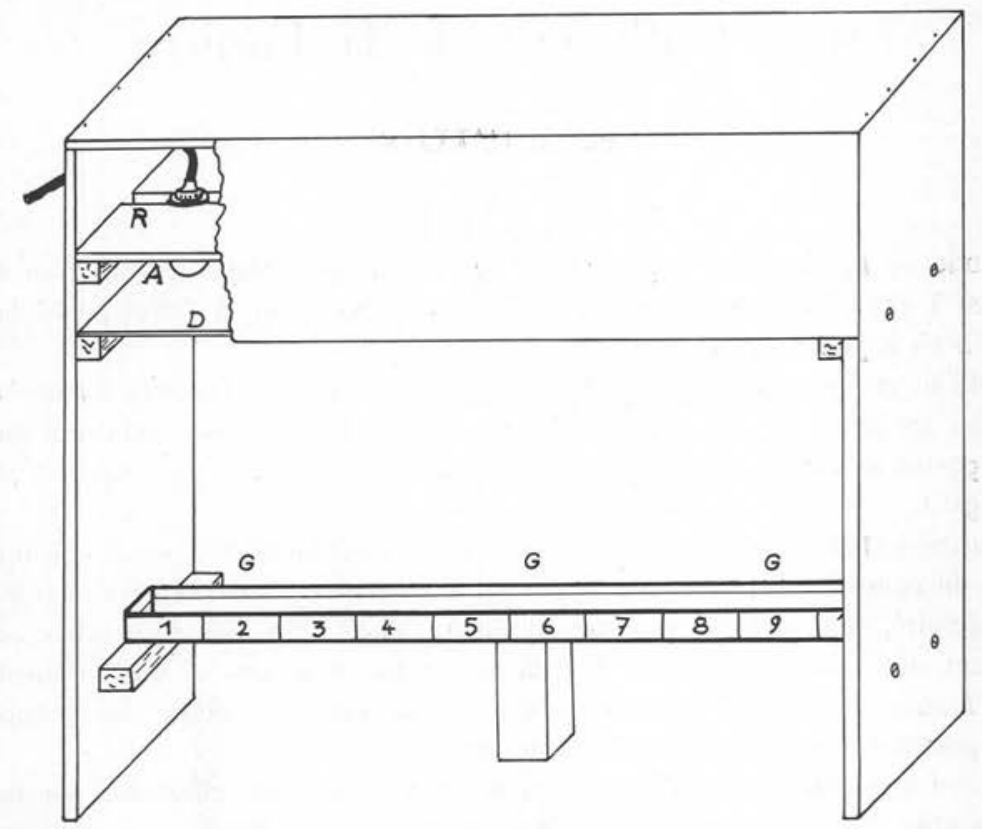

Fic. 1. - Schéma de l'appareil d'observation: A) une des ampoules; D) plaque de verre dépoli ; GG) gouttière de $1 \mathrm{~m} ; \mathrm{R})$ planchette avec une fente où peuvent glisser ou être fixées les ampoules

Les expériences ont toujours été conduites pour chaque espèce dans des conditions aussi identiques que possible d'éclairement, de température et de durée. L'appareil était placé dans une pièce obscure et, autant qu'il est possible, à l'abri de toute vibration.

L'observation est assez délicate et nécessite deux observateurs, c'est-à-dire un de chaque côté de la gouttière. Ils doivent opérer en évitant tout contact avec le dispositif et même être vêtus de sombre pour ne pas créer des éclairages intempestifs.

Les Culicides à examiner proviennent, soit d'élevages au laboratoire, soit de larves capturées dans la nature, suivant les espèces. 
Avant chaque mesure on isole 25 larves (ou nymphes) que l'on place dans le local où auront lieu les observations pour les amener, s'il y a lieu, à la température correspondant à l'eau de la gouttière qui a été remplie préalablement. Au moment de l'expérience les larves sont réparties délicatement dans la gouttière aussi régulièrement que possible dans toute sa longueur. L'éclairage est allumé et on compte les individus dans chaque case au bout de cinq, dix, quinze et vingt minutes. Avec ces quatre mesures sur 25 larves on a donc un résultat immédiat en pourcentage. Il y a intérêt à répéter les expériences le plus possible, mais pour ce faire, comme on ne peut pas se servir des mêmes individus, on rencontre des difficultés pour se procurer les larves de certaines espèces en assez grand nombre, au même stade, le même jour.

\section{RESULTATS}

\section{$1^{\circ}$ Aedes aegypti.}

La souche que nous avons utilisée était alors élevée depuis plus de douze ans au laboratoire. Elle provient originairement du laboratoire de Parasitologie de la Faculté de Médecine de Paris. Son origine exacte est confuse.
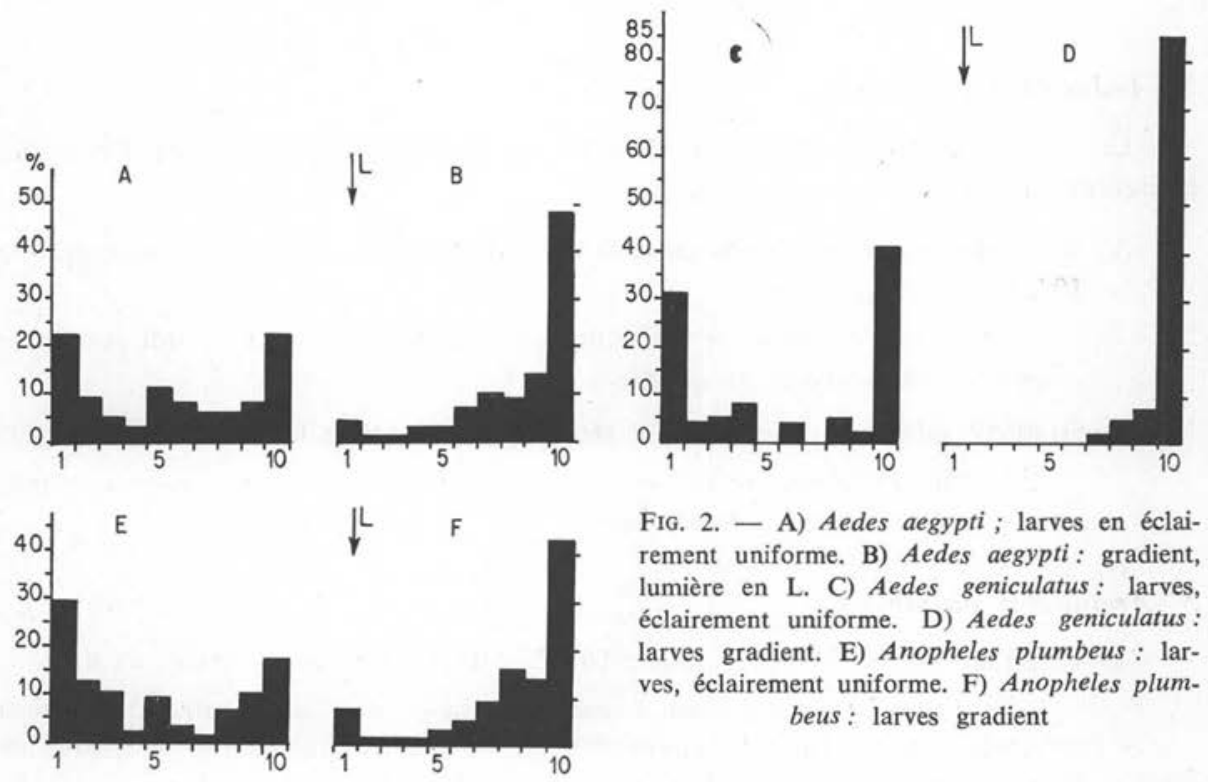

FIg. 2. - A) Aedes aegypti ; larves en éclairement uniforme. B) Aedes aegypti: gradient, lumière en L. C) Aedes geniculatus: larves, éclairement uniforme. D) Aedes geniculatus: larves gradient. E) Anopheles plumbeus : larves, éclairement uniforme. F) Anopheles plumbeus: larves gradient

A) Eclairage uniforme; température $22^{\circ} \mathrm{C}$. Quatre séries d'essais avec en tout 100 larves au $4^{\circ}$ stade. 
Les larves se disposent assez irrégulièrement dans la gouttière; elles se groupent assez volontiers aux deux extrémités, le long de la paroi, par un phénomène (thigmotactisme ?) assez fréquent chez plusieurs Culicidés (fig. 4).

B) Gradient ; mêmes conditions et même nombre ; larves au $4^{n}$ stade.

A part un certain nombre de larves qui se placent ou restent à l'extrémité la plus éclairée la grande majorité gagne l'extrémité la moins éclairée et on en trouve $48 \%$ dans la dernière case (fig. B).

Omardeen a observé un phototropisme négatif moins prononcé mais les larves que nous avons utilisées étaient certainement plus âgées en général que celles qu'il a testées et ainsi s'explique leur réaction, plus voisine de celle de la nymphe.

C) Eclairage uniforme; mêmes conditions ; 100 nymphes.

Elles se disposent indifféremment dans la gouttière, avec une prédilection pour les deux extrémités.

D) Gradient ; mêmes conditions ; 100 nymphes.

Les nymphes montrent un phototropisme négatif très net et se groupent, à part quelques individus, dans la case la moins éclairée. Là encore nous obtenons une réaction encore plus marquée que celle signalée par Omardeen, et peut-être pour les mêmes raisons.

\section{$2^{\circ}$ Aedes (F.) geniculatus.}

Les larves ont été récoltées dans divers trous d'arbres des environs de Strasbourg et élevées au laboratoire.

A) Eclairage uniforme ; larves au $4^{\circ}$ stade (50 larves en deux séries), température $19^{\circ}$.

$80 \%$ des individus se tiennent aux extrémités; les $20 \%$ qui restent se dispersent au centre de la gouttière (fig. C).

B) Gradient lumineux ; larves au $4^{\circ}$ stade ; 75 individus ; $19^{\circ} \mathrm{C}$.

Le phototropisme négatif est très net puisque $85 \%$ des individus se portent vers l'extrémité la moins éclairée (fig. D).

\section{$3^{\circ}$ Anopheles plumbeus.}

A) Eclairage uniforme ; température $16^{\circ} \mathrm{C}$; 50 larves en deux séries ; $4^{\circ}$ stade.

$29 \%$ des larves se portent à une extrémité et 16 à l'autre sans qu'on puisse déterminer pourquoi une extrémité a plus d'attraits que l'autre en dehors de la possibilité d'une répartition inégale au départ. Le reste des larves se répartit entre les autres cases (fig. E).

B) Gradient lumineux ; température $18-19^{\circ} \mathrm{C}$; même quantité de larves au $4^{\circ}$ stade. 
Le phototropisme négatif, déjà noté par Roubaud et Colas-Belcour, est manifeste mais cependant moins marqué qu'avec $A$. geniculatus (fig. F).

$4^{\circ}$ Culex pipiens souche autogène.

Cette souche provenait de Lyon et m'avait été communiquée par mon excellent collègue, le $\mathrm{P}^{\mathrm{r}} \mathrm{E}$. Roman. Elle était élevée depuis plus d'un an dans mon laboratoire. L'expérience a pu porter aisément sur 100 larves.

A) Eclairage uniforme. 100 larves au $4^{\circ}$ stade ; $22^{\circ} \mathrm{C}$.

Là encore on note le rassemblement d'une forte proportion des larves aux deux extrémités (19 et $22 \%$ ) et une répartition irrégulière avec un abaissement inexpliqué entre 8 et 9 (fig. G).
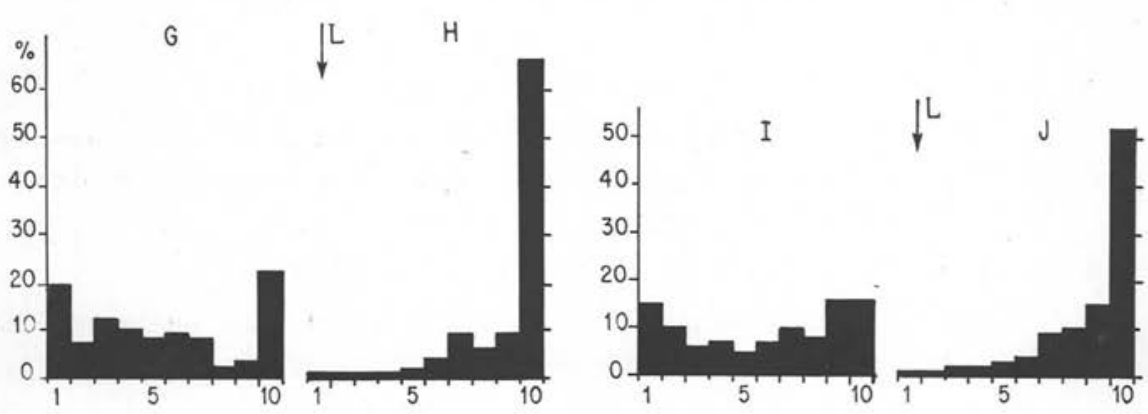

FIG. 3. - - G) Culex pipiens autogène; larves, éclairement uniforme. H) Culex autogène ; larves, gradient. I) Culex autogène; nymphes, éclairement uniforme. J) Culex autogène ; nymphes gradient. K) Culex pipiens fatigans; larves, éclairement uniforme. L) Culex pipiens fatigans, gradient

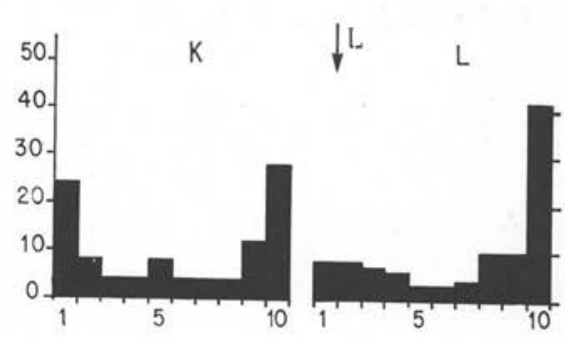

B) Gradient. Mêmes conditions et nombre.

Les larves se maintiennent dans la partie la moins éclairée de la gouttière de façon très nette (fig. $\mathrm{H}$ ).

C) La même expérience sur 50 nymphes a montré aussi une répartition uniforme (fig. I) avec l'éclairage uniforme et un phototropisme négatif net dans le gradient (fig. J). 
$5^{\circ}$ Culex pipiens pipiens souche rustique.

On a observé des larves récoltées dans une mare de forêt des environs de Stras. bourg.

Leur comportement dans des conditions identiques aux observations précédentes est assez voisin, mais le phototropisme négatif semble moins marqué :

\begin{tabular}{|r|r|r|r|r|r|r|r|r|r|r|r|}
\hline Cases .... & 1 & 2 & 3 & 4 & 5 & 6 & 7 & 8 & 9 & 10 \\
\cline { 1 - 3 } & \multirow{2}{*}{ Lumière $\rightarrow$} & 0 & 1 & 1 & 4 & 3 & 7 & 12 & 17 & 19 & 36 \\
\hline
\end{tabular}

\section{$6^{\circ}$ Culex pipiens fatigans.}

Cette souche provenait de Dakar et était élevée au laboratoire depuis quelques mois.

A) Eclairage uniforme ; température $22^{\circ} \mathrm{C}$; 100 larves.

La répartition se rapproche de celle qui a été décrite pour le Culex autogène ou Anopheles plumbeus, c'est-à-dire qu'un fort pourcentage de larves se porte vers les extrémités (fig. K).

B) Gradient. Mêmes conditions.

$41 \%$ des larves se portent vers la dernière case, mais une grande quantité se répartit entre les dernières et les premières cases (fig. L).

\section{$7^{\circ}$ Aedes (O.) communis.}

Cette espèce typique des mares temporaires de frêt dans les Vosges a été étudiée, du point de vue de son comportement, par Haufe (1957) au Canada dans les conditions naturelles, c'est-à-dire sous l'influence de la lumière et de la température.

A) Eclairage uniforme. 50 larves au $4^{\circ}$ stade ; $16^{\circ} \mathrm{C}$.

Des larves se portent aux deux extrémités ; des expériences plus nombreuses permettraient certainement d'éliminer les erreurs liées à la distribution primitive (fig. M).

B) Gradient ; 75 larves au $4^{\circ}$ stade ; $16^{\circ} \mathrm{C}$.

A part la case 1, où l'on trouve $11 \%$ des larves, les autres se portent graduellement mais régulièrement vers l'extrémité la moins éclairée (fig. $\mathrm{N}$ ).

\section{$8^{\circ}$ Aedes (O.) rusticus.}

La grande taille des larves permet une étude des stades plus jeunes facilement visibles. 
Les larves aux stades 2 et 3 se répartissent assez uniformément dans la gouttière uniformément éclairée. Avec les gradients lumineux cette disposition n'est guère modifiée.

\begin{tabular}{|r|r|r|r|r|r|r|r|r|r|r||}
\hline & $\mathbf{L} \downarrow$ \\
\hline Cases ... & \multicolumn{8}{|c|}{ Aedes rusticus stade 2 et 3} \\
\hline Larves 2 . & 1 & 2 & 3 & 4 & 5 & 6 & 7 & 8 & 9 & 10 \\
\hline Larves 3. & 5 & 17 & 11 & 5 & 13 & 12 & 9 & 3 & 5 & 16 \\
\hline
\end{tabular}

Au quatrième stade, alors que la répartition paraît sans signification en lumière uniforme (fig. O), il y a, avec le gradient, une nette accumulation des larves dans la partie centrale de la gouttière (fig. P).

\section{$9^{\circ}$ Culex (N.) hortensis.}

La répartition des larves au quatrième stade se fait au hasard en éclairage uniforme et avec un gradient lumineux la répartition n'est guère modifiée (fig. Q). Les
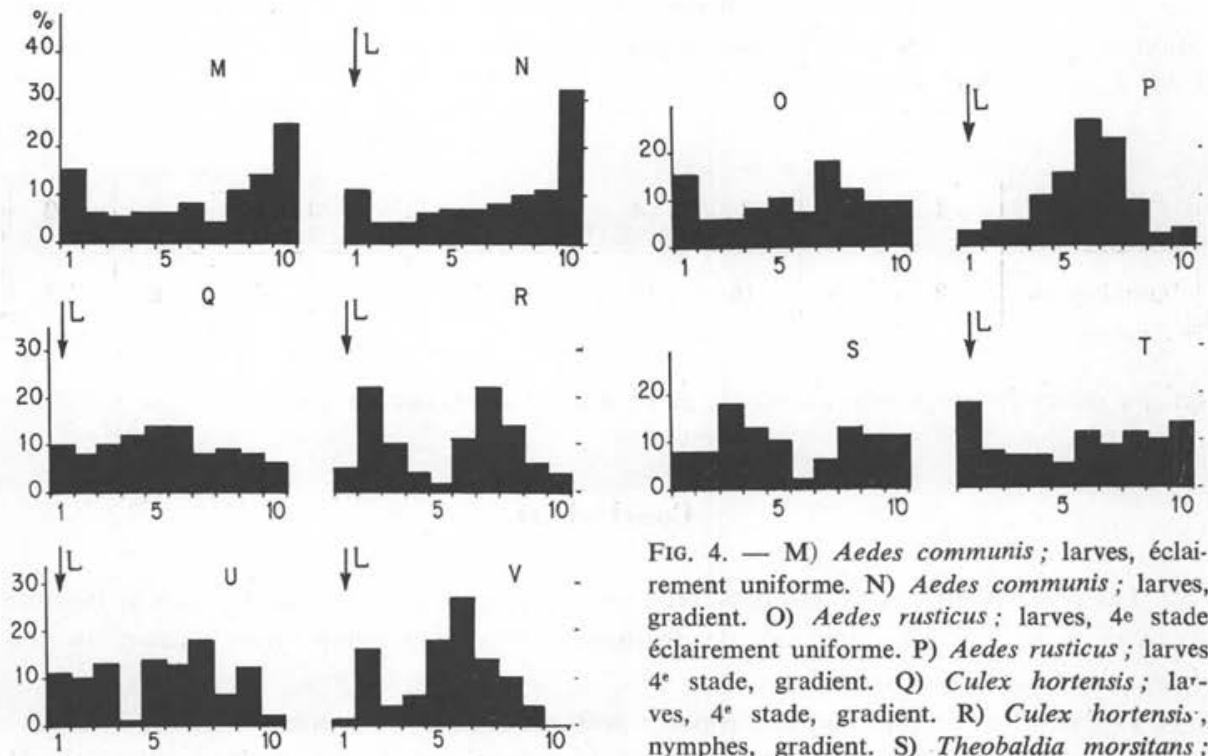

FIG. 4. - M) Aedes communis; larves, éclairement uniforme. N) Aedes communis; larves, gradient. O) Aedes rusticus; larves, 4e stade éclairement uniforme. P) Aedes rusticus; larves $4^{\text {e }}$ stade, gradient. Q) Culex hortensis; larves, 4" stade, gradient. R) Culex hortensis; nymphes, gradient. S) Theobaldia morsitans; larves, éclairement uniforme. T) Theobaldia morsitans; larves, gradient. U) Anopheles claviger; larves. gradient. V) Anopheles maculipennis messeae; larves gradient 
nymphes, mais observées en petit nombre (50), se sont groupées sous le gradient en deux zones qui ne s'expliquent, ni par l'éclairement, ni par un échauffement progressif, qui n’a pas été constaté du reste (fig. R).

\section{$10^{\circ}$ Theobaldia (C.) morsitans.}

Les larves au $4^{\circ}$ stade ont été observées en milieu peut-être trop chaud $\left(20^{\circ} \mathrm{C}\right)$; en lumière uniforme leur répartition est irrégulière (fig. S) et sous le gradient elle se fait assez uniformément mais reflète plus l'immobilité de ses larves qui restent volontiers immergées sur le fond (fig. T).

\section{$11^{\circ}$ Anopheles (A.) claviger.}

La distribution des individus étudiés en trop petit nombre : 25 larves, semble liée à la répartition initiale dans la gouttière; les larves restent en effet immobiles (fig. U).

\section{$12^{\circ}$ Anopheles maculipennis messeae.}

Notre expérience est trop restreinte, mais un essai avec seulement 25 larves avait montré sous le gradient une distribution peut-être due à la répartition initiale (fig. V).

\section{$13^{\circ}$ Mochlonyx culiciformis.}

Les larves de ce prédateur sont toujours en mouvement de va-et-vient et leur répartition est difficile à enregistrer. Sous le gradient nous avons eu, avec 25 larves observées à $16^{\circ} \mathrm{C}$, le résultat suivant :

\begin{tabular}{|r|r|r|r|r|r|r|r|r|r|r||}
\hline \hline Cases .... & 1 & 2 & 3 & 4 & 5 & 6 & 7 & 8 & 9 & 10 \\
\hline Lumière $\rightarrow$ & 8 & 16 & 16 & 12 & 10 & 13 & 6 & 7 & 6 & 6 \\
\hline
\end{tabular}

qui ne différait pas sensiblement de celui obtenu en éclairage uniforme.

\section{Conclusions}

Les résultats de ces expériences, très incomplètes et très insuffisantes par le nombre d'observations, permet cependant de distinguer, selon leur comportement dans un gradient, deux catégories de larves.

D'abord un certain nombre d'espèces présentant un phototropisme négatif bien net : Aedes aegypti, Aedes geniculatus, Anopheles plumbeus, Culex pipiens autogène, $C$. pipiens pipiens, $C$. pipiens fatigans. 
Il est à remarquer qu'il s'agit d'espèces de gîtes souvent peu éclairés, voire obscurs, car hypogés.

A un degré moindre Aedes communis, qui a un comportement assez particulier dans la nature (Haufe), présente encore un phototropisme négatif net.

Par contre les autres espèces étudiées, comme Aedes rusticus, Culex hortensis, Theobaldia morsitans, etc., n'ont pas paru marquer de préférence spéciale pour la région la moins éclairée, or parmi celles-ci $C$. hortensis est une espèce rencontrée très fréquemment dans des gîtes très éclairés.

On a beau savoir que ce ne sont pas les larves qui choisissent leurs gîtes mais les femelles qui viennent y déposer leurs œufs, on n'en est pas moins frappé par les correspondances, objectivées par ces quelques observations sommaires, avec le comportement des larves et l'éclairement naturel de certains gîtes.

\section{Bibliographie}

BATES (M.), 1949. - The natural history of mosquitoes, New York, p. 120-122.

Haufe (W. O), 1957. - Physical environment and behaviour of immature stages of Aedes communis (Deg.) (Diptera: Culicidae) in subarctic Canada. Canad. Entomol., 89, 120-139.

OMARDEEn (T. A.), 1958. - The behaviour of larvae and pupae of Aedes aegypti (L) in light and temperature gradients. Bull. Ent. Res., 48, 349-357.

Roubaud (E.) et Colas-Belcour (J.), 1932. - Observations sur la biologie de l'Anopheles plumbeus. I. Le comportement larvaire. Bull. Soc. Path. Exot., 25, 763-770.

(Institut de Parasitologie, Faculté de Médecine, Strasbourg) 